\title{
Development and evaluation of a safe and effective sugar-free herbal lollipop that kills cavity-causing bacteria
}

\author{
Chu-hong $\mathrm{Hu}^{1}$, Jian $\mathrm{He}^{1}$, Randal Eckert ${ }^{2}$, Xiao-yang $\mathrm{Wu}^{1}$, Li-na $\mathrm{Li}^{1}$, Yan Tian ${ }^{1}$, Renate Lux ${ }^{1}$, Justin A. Shuffer ${ }^{1}$, Faina \\ Gelman $^{1}$, Janet Mentes ${ }^{3}$, Sue Spackman ${ }^{1}$, Janet Bauer ${ }^{1}$, Maxwell H. Anderson ${ }^{2}$, Wen-yuan Shi ${ }^{1}$ * \\ ${ }^{I}$ School of Dentistry, University of California, Los Angeles, USA; ${ }^{2}$ C3 Jian Inc, Inglewood, USA; ${ }^{3}$ UCLA School of \\ Nursing, University of California, Los Angeles, USA
}

Dental caries (tooth decay) is caused by a specific group of cariogenic bacteria, like Streptococcus mutans, which convert dietary sugars into acids that dissolve the mineral in tooth structure. Killing cariogenic bacteria is an effective way to control or prevent tooth decay. In a previous study, we discovered a novel compound (Glycyrrhizol A), from the extraction of licorice roots, with strong antimicrobial activity against cariogenic bacteria. In the current study, we developed a method to produce these specific herbal extracts in large quantities, and then used these extracts to develop a sugar-free lollipop that effectively kills cariogenic bacteria like Streptococcus mutans. Further studies showed that these sugar-free lollipops are safe and their antimicrobial activity is stable. Two pilot human studies indicate that a brief application of these lollipops (twice a day for ten days) led to a marked reduction of cariogenic bacteria in oral cavity among most human subjects tested. This herbal lollipop could be a novel tool to promote oral health through functional foods.

Keywords: antimicrobial therapy; licorice; Streptococcus mutans

International Journal of Oral Science (2011) 3: 13-20. doi: 10.4248/IJOS11005

\section{Introduction}

Dental caries (tooth decay) is a highly prevalent and costly disease in the United States and throughout the world. A recent Surgeon General's report showed that $45 \%$ of children aged 5-17 in the United States have varying levels of dental caries, and the report noted severe disease risk among children in underserved populations, such as Hispanic Americans and Native Americans [1]. Tooth decay is also increasing in incidence in the elder population of the United States [2].

Dental caries is an infectious disease dependent on a few species of cariogenic (cavity-causing) bacteria and specific dietary patterns (such as sugar intake and eating

*Correspondence: Wen-yuan Shi

Tel: 001310825 8356; Fax: 0013107947109

E-mail: wenyuan@ucla.edu

Received 8 August 2010; Accepted 6 September 2010 frequency) [3-7]. Streptococcus mutans (S. mutans), Streptococcus sobrinus (S. sobrinus), and Lactobacillus casei (L. casei) are some of the well-known cavity-causing bacteria, while $S$. mutans is the major contributor to acid production [8-10]. Most cavity-causing bacteria colonize tooth surfaces and produce acids that dissolve tooth structures. Repeated dissolution events lead to cavitation and the subsequent need for repair of the damaged areas [11-13]. Given the fact that dental caries is an infectious disease, antimicrobial treatments against cavity-causing bacteria should help control or prevent tooth decay. Indeed, previous studies have shown that the applications of various antimicrobial agents to the oral cavity can reduce tooth decay. In this study, we investigated a licorice extract for its ability to kill cavity-causing bacteria.

Licorice is the name applied to the roots and stolons of Glycyrrhiza species [14]. In a previous study, we generated a herbal extract from the roots of Glycyrrhiza uralensis, and the extract showed strong bacteriocidal 
effects against cavity-causing bacteria like S. mutans. Further studies led to the discovery of a novel compound, Glycyrrhizol A, which is responsible for this bio-activity [15]. In the current study, we aimed to produce the effective herbal extract in large quantity, and use this extract to develop a sugar-free lollipop that could effectively kill cavity-causing bacteria like $S$. mutans in vitro. We then ran safety, toxicity, stability and in vivo efficacy studies to verify the usefulness of this lollipop in killing cavity-causing bacteria in humans.

\section{Materials and Methods}

\section{Production of effective licorice extracts in large quantity} $100 \mathrm{~kg}$ of licorice roots were cut into thin slices, soaked with $95 \%$ food grade ethanol in a $1: 8$ ratio in extraction containers, and incubated at $37{ }^{\circ} \mathrm{C}$ for $72 \mathrm{~h}$. The resulting ethanol solutions (containing extracts) were filtered three times. The extracts were then concentrated through ethanol evaporation and dried with a microwave "dry" distillation method, which is a combination of microwave heating and dry distillation, performed at atmospheric pressure.

\section{Chemical analysis of glycyrrhizol A within licorice extract with high performance liquid chromatography}

The quality of the herbal extracts was assayed with high performance liquid chromatography (HPLC, Amersham Biosciences, Sweden) for the amount of Glycyrrhizol A. The HPLC conditions were: column, SOURCETM 15RPC (4.6/100); flow rate, $0.5 \mathrm{~mL} \cdot \mathrm{min}^{-1}$; elution system, $\mathrm{H}_{2} \mathrm{O}(0.1 \%$ TFA, solvent $\mathrm{A})$ and $\mathrm{CH}_{3} \mathrm{CN}(0.1 \%$ TFA, solvent B); detector, UV 215, 260 and $280 \mathrm{~nm}$; eluting gradient, from $10 \%$ (B) to $100 \%$ (B) in $15 \mathrm{CV}$ (column volume, $1 \mathrm{CV}=1.662 \mathrm{~mL}$ ).

\section{Analysis of residual pesticides, lead and arsenic within licorice extracts}

These studies were performed by Michelson Laboratories, Inc. The detailed protocols can be found at http://www.michelsonlab.com.

\section{Bacterial strains and culture growth conditions}

The following cavity-causing bacteria were used in the study: S. utans, strain UA159; S. sobrinus, strain ATCC27607; and, L. casei, strain ATCC334. All bacteria were grown at $37{ }^{\circ} \mathrm{C}$ in brain-heart infusion broth (BHI, Fisher) under anaerobic contions $\left(90 \% \mathrm{~N}_{2}, 5 \% \mathrm{CO}_{2}\right.$, $5 \% \mathrm{H}_{2}$ ).

\section{Determination of minimum inhibitory concentrations}

The antimicrobial activity of the licorice extract was first tested by microdilution broth assay based on the Clinical and Laboratory Standards Institute (CLSI) recommended protocol. In general, an over-night bacterial culture was diluted to $\sim 10^{5} \mathrm{cfu} \cdot \mathrm{mL}^{-1}$ with brain-heart infusion (BHI) and placed in a 96-well plate. Two-fold serial dilutions of licorice extracts were added to the 96-well plate seeded with diluted bacteria and incubated for $18-24 \mathrm{~h}$ at $37{ }^{\circ} \mathrm{C}$ under anaerobic conditions. The minimum inhibitory concentration (MIC) was determined as the concentration of licorice presenting in the first clear well.

Creation of sugar-free herbal lollipop with licorice extracts

A standard sugar-free candy formula from Dr. John's Candy (http://www.drjohns.com/) was used for making the herbal lollipop. The main ingredients were: hydrogenated starch hydrolysate (HSH) (solidifying agent), citric acid and mint (flavoring agents), FD \& C blue \#1,2; Red 3, 40; Yellow 5, 6 (coloring agents), and acesulfame potassium (noncaloric sweetener). Depending on the concentration of Glycyrrhizol A in a batch of licorice extract, 7 to $15 \mathrm{mg}$ of licorice extracts were added to each lollipop to achieve a uniform concentration of Glycyrrhizol A.

The lollipop making process involves various temperatures from $135{ }^{\circ} \mathrm{C}$ cooked syrup to $65{ }^{\circ} \mathrm{C}$ on the cooling table. Thermal stability of Glycyrrhizol A at various temperatures was tested and herbal extracts were added at temperatures that did not affect their bioactivities.

Testing bioactivity of herbal lollipops against cavitycausing bacteria

The bioactivities of herbal lollipops against cavitycausing bacteria were analyzed with two assays: overnight growth inhibition and time killing assay.

For the overnight growth inhibition study, a lollipop ( $\sim 10 \mathrm{~g} /$ piece) was broken into powder by a high speed pulverizing machine and dissolved in $10 \mathrm{~mL}$ BHI broth, then mixed with a bacterial testing solution $1: 1$ to final $\mathrm{OD}_{600}=0.1$. After 12 hours of incubation, $\mathrm{OD}_{600}$ was measured again to indicate the inhibitory effects against bacterial growth.

For the time killing assay, solutions derived from the herbal lollipop and the placebo lollipop were prepared. A cariogenic $S$. mutans strain was grown into log phase under anaerobic conditions overnight, and diluted with BHI growth medium to $\sim 10^{6} \mathrm{~mL}^{-1}$. The bacterial culture was then mixed with the different solutions described above at a ratio $1: 10$ and incubated for 1,5 , and $10 \mathrm{~min}$, respectively. The incubated mixtures were rescued in large volume of BHI broth, and then plated on BHI agar 
plates, incubated overnight at $37{ }^{\circ} \mathrm{C}$, and counted for surviving bacteria.

\section{Studying the stability of the herbal lollipops}

The herbal lollipops were placed at 25,37 , and $60{ }^{\circ} \mathrm{C}$ incubators for various times and then examined for their antimicrobial activities against cariogenic bacteria using the overnight growth inhibition and time killing assays described above.

\section{Studying the safety of the herbal lollipops}

Genotoxicity of licorice extract was examined with the Ames tests [16] using Salmonella typhimurium TA 1535 (His deficiency) (ATCC 29629), TA1537 (His deficiency) (ATCC 29630), and Escherichia coli WP2 uvrA (Trp deficiency) (ATCC 49979).

Cytotoxicity of licorice extract was examined with Promega's CellTiterGlo assay using various epithelial and immune cell lines: Jurkat, HOK68, CHO and BHK.

The safety of the licorice extract was also examined with single dose acute toxicity study with mice. In these studies, licorice extract was orally administered via pipette for $45 \mathrm{~s}$ for each mouse (holding the neck to reduce swallowing). Each mouse received $3 \mathrm{mg}$ in a $100 \mu \mathrm{L}$ volume in $20 \mu \mathrm{L}$ increments, which they would swallow intermittently. The $45 \mathrm{~s}$ treatment time refers to the time that mice harbored a visible volume of licorice extract prior to swallowing. Mice were visually observed for oral irritation changes of behavior daily following treatment. Mice were also weighed weekly for aberrant weight fluctuations. Two weeks post-treatment, the mice were euthanized for pathological examination on major organs (heart, liver, spleen, intestines, stomach, kidney and lung).

\section{Protocols for pilot human studies}

Two pilot human studies were authorized by UCLA IRB committee and performed using identical protocols. The two studies were of 10-day duration, involving a total of 26 subjects at different ages. There were two study visits, including the initial screening/baseline visit over the 10-day study period (baseline and 10 days). All visits were scheduled in the morning before lunch.

At the baseline visit, after affirmative completion of the informed consent process and all necessary forms, each subject underwent a visual and photographic clinical oral examination. For those subjects who visually met the study criteria, a saliva sample was obtained. At total of $0.5 \mathrm{~mL}$ of saliva was collected and placed in the provided $1.5 \mathrm{~mL}$ Eppendorf tube. These samples were used to measure the amount of $S$. mutans present, using the monoclonal antibody based $S$. mutans detection method described below [17]. To qualify for the study, subjects had to have a minimum of $1.0 \times 10^{5} \mathrm{~S}$. mutans cells $/ \mathrm{mL}$ in their saliva samples.

Once the bacterial samples (saliva) were analyzed, we notified all subjects of their qualification status. Qualified subjects were given oral and written product use instructions. All human subjects were provided with 20 lollipops. After using the products for 10 days, the subjects attended a morning appointment. Subjects were asked about any adverse events they may have experienced since the previous visit. They then provided samples of their saliva and a visual examination of their mouth was performed to assess the status of the oral tissues. Data were analyzed using various statistical methods, as presented in the Results.

Analysis of salivary S. mutans using species-specific monoclonal antibody

At total of $10 \mathrm{~mL}$ of saliva sample fixed with $1 \%$ formaldehyde was incubated with $10 \mathrm{~mL}$ purified antiS. mutans monoclonal antibody for $30 \mathrm{~min}$, and then $0.5 \mathrm{~mL}$ FITC labeled anti-mouse IgG (Sigma, USA) was added to the mixture and incubated for another $1 \mathrm{~h}$. $10 \mathrm{~mL}$ of the above mixed sample was then checked under a florescent microscope (Nikon eclipse E400, Japan).

\section{Results}

Producing large quantity licorice extracts with strong antimicrobial activity against cavity-causing bacteria

Following our previous discovery [15] that a specific licorice extract has strong antimicrobial activity against cavity-causing bacteria due to the existence of a highly potent antimicrobial compound, Glycyrrhizol A, we aimed to develop a sugar-free candy supplemented with licorice extracts to promote oral health. In order to achieve this goal, we first needed to produce effective licorice extracts in large quantity, and in a safe manner.

In collaboration with a Chinese manufacturer specialized in herbal extraction, we collected five different licorice root samples harvested from different parts of China (Zhejiang, Jiangsu, Gansu, Xingjiang and Inner Mongolia provinces), cut licorice roots into different shapes (thin slices, small rods, or ground powders), and used different extraction methods (such as water boiling or ethanol soaking) for different periods of time. Most importantly, instead of using toxic organic solvents described in the previous paper [15], we successfully developed a new protocol to achieve the same effect by using food-grade ethanol.

The licorice extract produced in large quantity was 
chemically analyzed with HPLC. As shown in Figure 1, the extract did contain high levels of Glycyrrhizol A. Additional chemical analyses indicated that the extract had no detectable pesticides, lead, orarsenic (Table 1). We examined the anti-microbial activity of this large- scale prepared licorice extract. As shown in Table 2, the extract had strong antimicrobial activity against the major cavity-causing bacteria, as the MIC against these bacteria are less than $40 \mu \mathrm{g} \cdot \mathrm{mL}^{-1}$.

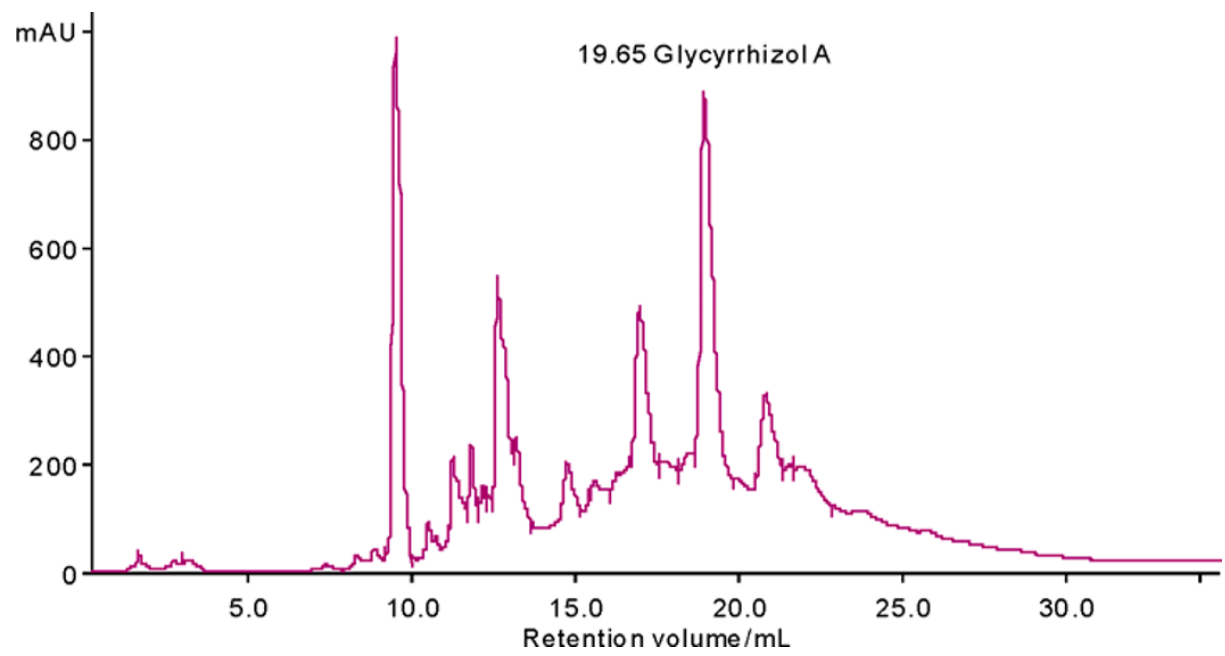

Figure 1 HPLC analysis of Glycyrrhizol A within licorice extract. Glycyrrhizol A is detected by Amersham Biosciences HPLC with UV 215 at retention volume $19.65 \mathrm{~mL}$.

Table 1 Analysis of hazard chemicals within the licorice extract (performed by Michelson Laboratories, Commerce, CA)

\begin{tabular}{ll}
\hline \multicolumn{1}{c}{ Testing item } & Results \\
\hline Lead & Not detected \\
Arsenic & Not detected \\
Organo-halide pesticides & Not detected \\
Organo-phosphate pesticides & Not detected \\
Carbamate pesticides & Not detected \\
\hline
\end{tabular}

Table 2 MIC of large scale prepared herbal extracts against major cavity-causing bacteria

\begin{tabular}{lc}
\hline Tested cavity-causing bacteria & MIC* \\
\hline S. mutans & 15.6 \\
S. sobrinus & 31.2 \\
L. casei & 31.2 \\
\hline
\end{tabular}

*MIC: represents average of at least three independent experiments.

Developing a sugar-free herbal lollipop with bactericidal effects against cavity-causing bacteria

With large-scale production of effective herbal extracts accomplished, we began to consider the best methods to deliver this active antimicrobial herbal extract to the oral cavity. After extensive discussion, we decided to create a sugar-free candy, supplemented with the herbal extract as the carrier to deliver the naturally occurring anti- microbial component. The rationale was that we could deliver the antimicrobial components through the same mechanism that causes the problem of dental caries, i.e. use a behavioral pattern that causes the problem (candy consumption) to solve the problem. This strategy allows access to the at-risk population without demanding behavior changes. Among different forms of candies, we chose lollipops, since they are the acceptable and safe delivery mechanisms welcomed by children, seniors, and other needy populations, such as the severely disabled. Furthermore, the act of sucking on lollipops helps stimulate salivary production to advantage remineralization, and because of the volume and dissolution rate, the teeth get maximum exposure time to the herbal extracts. This adds substantivity to the delivery system since it takes longer (10-15 $\mathrm{min})$ to dissolve a lollipop compared with other candy forms we tested.

In collaboration with Dr. John's Candies, we went through a number of different formulations and production procedures to develop a formula in which the herbal extracts were mixed with candy syrup at a temperature and duration that does minimal damage to their bioactivity, and ensured that a sufficient amount of herbal extract was added to each lollipop to guarantee its bioactivity. The currently marketed lollipop is the finished product of this study. It is worthwhile noting that, depending on the concentration of the active ingredient in different batches, herbal extracts have MICs against 
the major cavity-causing bacteria ranging from 13$40 \mu \mathrm{g} \cdot \mathrm{mL}^{-1}$. After testing, an adjusted amount of herbal extracts (from $7-15 \mathrm{mg}$ ) are added to each lollipop to ensure final herbal concentrations in oral cavity are around $25 \times$ higher than the MIC, since it takes about 10-15 min (with the production of $10-15 \mathrm{~mL}$ saliva) [7] to dissolve a lollipop (about $10 \mathrm{~g}$ ). The actual data on the efficacy and stability of the herbal lollipops are described below.

Studying in vitro efficacy and stability of herbal lollipops

The in vitro antimicrobial activity of the herbal lollipop was first assayed with an overnight growth inhibition assay. As shown in Table 3, herbal lollipops exhibited good antimicrobial activities against the measured cavity-causing bacteria, indicating that herbal extracts embedded in the lollipops retained their antimicrobial bioactivities.

Since these data were obtained by incubating the bacteria with lollipop solutions overnight, while a lollipop dissolves in the oral cavity in $10-15 \mathrm{~min}$, a direct comparison study was performed to determine if exposure to an herbal lollipop would kill the cavity-causing bacteria in 10-15 min. Given a stimulated human salivary flow rate of approximately $1 \mathrm{~mL} \cdot \mathrm{min}^{-1}$ [7], a lollipop was dissolved in a $10-15 \mathrm{~mL}$ solution and tested for its bactericidal activity after $15 \mathrm{~min}$, in comparison with placebo lollipops. As shown in Figure 2, the herbal lollipop exhibited significantly better antimicrobial activities against cavity-causing bacteria compared to the placebo lollipops.

Both the overnight growth inhibition and time killing assays are routinely used to test each freshly made batch of herbal lollipops to ensure their quality.

We also ran an extensive stability study of the herbal lollipops by placing them at 25,37 , and $60{ }^{\circ} \mathrm{C}$ incubators for various times and then examined their antimicrobial activities against cavity-causing bacteria using both the overnight growth inhibition and time killing assays described above. No significant reduction in bioactivities was observed in nine months.

\section{Examining the safety of the herbal lollipop}

All ingredients used in the sugar-free lollipops are FDA-approved for human consumption. Licorice has been used by various cultures for thousands of years, and many previous studies have shown that it is a safe medicinal herb [18-19]. FDA lists licorice as GRAS (generally regarded as safe) when used as a food flavoring or sweetening agent.

Table 3 Antimicrobial activity of the herbal lollipop

\begin{tabular}{ccc}
\hline Tested cavity-causing bacteria & $\mathrm{OD}_{600}$ after $12 \mathrm{~h}$ with placebo lollipop & $\mathrm{OD}_{600}$ after $12 \mathrm{~h}$ with herbal lollipop \\
\hline S. mutans & 1.5 & 0.1 \\
S. sobrinus & 1.7 & 0.1 \\
L. casei & 1.4 & 0.1 \\
\hline
\end{tabular}

Lollipop $(\sim 10 \mathrm{~g})$ with or without licorice extract is dissolved in $10 \mathrm{~mL}$ solution and mixed with bacterial solution $1: 1$. Initial $\mathrm{OD}_{600}$ reading is 0.1 .

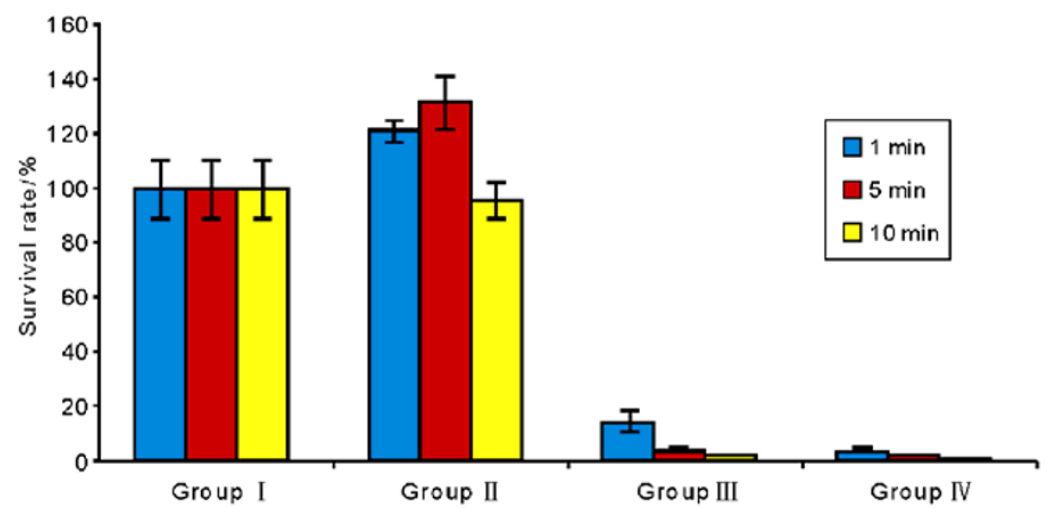

Figure 2 Time killing assay. Bioactivity of lollipops against cavity-causing bacteria $S$. mutans planktonic cells. Diluted S. mutans were exposed to TH broth (group I), $0.125 \mathrm{~g} \cdot \mathrm{mL}^{-1}$ lollipop placebo (group II), $0.25 \mathrm{~g} \cdot \mathrm{mL}^{-1} \mathrm{herbal}$ Iollipop (group III) and $1 \mathrm{~g} \cdot \mathrm{mL}^{-1}$ herbal lollipop (group IV) for 1, 5, and $10 \mathrm{~min}$. Survival rates (\%) were calculated by dividing surviving CFU per milliliter of each lollipop solution by surviving CFU per milliliter of $\mathrm{TH}$ broth. Data represents averages of the results of three independent experiments. 
Table 4 Summary of toxicity/safety studies on the licorice extract

\begin{tabular}{lc}
\hline \multicolumn{1}{c}{ The performed toxicity/safety tests } & \multicolumn{1}{c}{ The results } \\
\hline Ames tests using S. typhimurium TA1535 (His deficiency) (ATCC 29629), S. & No genotoxicity was detected \\
typhimurium TA1537 (His deficiency) (ATCC 29630) and Escherichia coli WP2 & \\
uvrA (Trp deficiency) (ATCC 49979) & \\
Cytotoxicity study with Promega's CellTiterGlo assay using cell lines of Jurkat, & No cellular toxicity was detected \\
HOK68, CHO and BHK & \\
Single dose acute toxicity study with mice on oral irritation, behavioral changes, and & No animal toxicity was detected \\
pathological examination on major organs (heart, liver, spleen, intestines, stomach, & \\
kidney, and lung)
\end{tabular}

Table 5 Pilot human study: salivary S. mutans analysis before and after herbal lollipop treatment

\begin{tabular}{ccc||ccc}
\hline Subjects & Baseline & After treatment & Subjects & Baseline & After treatment \\
\hline 1 & $6.67 \times 10^{5}$ & $1.33 \times 10^{6}$ & 14 & $3.70 \times 10^{7}$ & $3.82 \times 10^{7}$ \\
2 & $1.67 \times 10^{6}$ & $4.00 \times 10^{5}$ & 15 & $2.00 \times 10^{6}$ & $1.24 \times 10^{5}$ \\
3 & $3.33 \times 10^{5}$ & $3.33 \times 10^{5}$ & 16 & $7.40 \times 10^{5}$ & $2.40 \times 10^{7}$ \\
4 & $3.00 \times 10^{5}$ & $1.33 \times 10^{5}$ & 17 & $1.80 \times 10^{5}$ & $1.35 \times 10^{5}$ \\
5 & $2.33 \times 10^{5}$ & $1.00 \times 10^{5}$ & 18 & 0.00 & $2.68 \times 10^{7}$ \\
6 & $1.00 \times 10^{5}$ & $1.33 \times 10^{4}$ & 19 & $1.30 \times 10^{6}$ & $2.51 \times 10^{5}$ \\
7 & $6.00 \times 10^{5}$ & $3.33 \times 10^{4}$ & 20 & $2.30 \times 10^{7}$ & $2.02 \times 10^{5}$ \\
8 & $4.26 \times 10^{7}$ & $1.00 \times 10^{4}$ & 21 & $1.00 \times 10^{7}$ & $1.10 \times 10^{5}$ \\
9 & $3.00 \times 10^{7}$ & $1.44 \times 10^{5}$ & 22 & $4.09 \times 10^{7}$ & $2.91 \times 10^{5}$ \\
10 & $5.00 \times 10^{7}$ & $9.12 \times 10^{4}$ & 23 & $1.00 \times 10^{7}$ & $2.26 \times 10^{5}$ \\
11 & $3.70 \times 10^{6}$ & 0.00 & 24 & $1.00 \times 10^{7}$ & $1.00 \times 10^{5}$ \\
12 & $1.48 \times 10^{6}$ & $2.40 \times 10^{5}$ & 25 & $3.72 \times 10^{5}$ & $1.40 \times 10^{5}$ \\
13 & $8.00 \times 10^{5}$ & $1.71 \times 10^{5}$ & 26 & $1.24 \times 10^{5}$ & 0.00 \\
\hline
\end{tabular}

All numbers represent $S$. mutans counts in $1 \mathrm{~mL}$ saliva. 0.00 is a statistical readout which indicates $S$. mutans is undetectable due to current experimental method limitation.

Since the original raw data do not distribute normally, the statistical analysis was performed on log scale with proc $t$-test using SAS version 9 (SAS Institute Inc., Cary, NC). $P$-value for testing difference (Normally distributed): 0.853 5; Mean difference: $\log$ (after) - $\log$ (before): -2.41; Standard error of mean difference: 0.5497 ; $t$-test statistic: -4.38 ; Number of before/after pairs both having values: $23 ; P$-value for testing mean difference is $0: 0.0002$.

We performed additional safety tests on the herbal lollipops, including the Ames test based genotoxicity study, CellTiterGlo based cytotoxicity studies, and acute animal toxicity studies, as described in the Materials and Methods. As shown in Table 4, all toxicity studies had negative outcomes, further confirming the safety of this herbal lollipop.

Verifying the in vivo efficacy of herbal lollipop for its ability to kill cavity-causing bacteria in human oral cavity

Approved by the UCLA IRB committee, we performed two pilot human studies at UCLA Children's Dental Clinic (20 subjects) and two Los Angeles nursing homes (6 subjects). Due to the limited number of human subjects, no control groups were used for these initial studies. Instead, all subjects were initially tested for salivary S. mutans using a monoclonal antibody based test [17], then exposed to the lollipops for ten days (twice a day) before repeat testing for salivary $S$. mutans. As shown in Table 5, and consistent with in vitro studies, a majority of subjects exhibited dramatic reductions of salivary $S$. mutans after using the lollipop.

\section{Discussion}

This study describes the development of an herbal lollipop that can kill cavity-causing bacteria. The studies showed that these sugar-free lollipops are safe and their antimicrobial activities are stable in the formulations intended for delivery. Two pilot human studies indicated that a brief application of these lollipops (twice a day for 10 days) led to marked reduction of cavity-causing bacteria in oral cavity among most of the human subjects 
tested [20-21]. We recognize that while the data from pilot human studies are encouraging, they are preliminary. In the future, we plan to design double-blind, randomized, longitudinal studies that include more human subjects and more examination points, to give us better data about the lollipop's long term efficacy. We also plan to do more detailed studies on how soon the cavitycausing bacteria grow back after treatment, why there are variable results among some people, and how frequently the herbal lollipops should be used to achieve maximum protective effects. From the basic science perspective, we are interested in learning the killing mechanism(s) by which Glycyrrhizol A exerts its effects and how likely a cavity-causing bacterial cell is to develop the resistance against the compound. We specifically applied the herbal lollipop in a dosing regimen (twice per day for 10 days) designed to preclude the development of resistant forms. The regimen was also structured to minimize the costs to purchasing entities, so that this system might be used in public health settings. Rather than applying the herbal lollipop as a low-dose, long-term strategy (increasing cost and the opportunity for resistance), a short-term, intensive strategy was employed. This is consistent with the evolving science concerning biofilms and their adaptation to metabolic and antimicrobial pressures.

Previous studies have shown a clear connection between killing cavity-causing bacteria and reductions in tooth decay. Since the killing effect against cavitycausing bacteria of this herbal lollipop is evident, use of this lollipop may lead to control or prevention of tooth decay, making it a novel tool to promote oral health as a functional food for special dietary use. However, we still need to demonstrate this anticavity effect clinically, rather than just via the microbiology end-point.

In our preliminary studies, we found that licorice extract did not interfere with the activity of other anticavity components such as fluoride and xylitol. It may be interesting to study the possible synergistic effects between and amongst these ingredients. Lollipops turn out to be a useful delivery device welcomed by different populations with problems in oral cavity. One could consider using it as a delivery device to treat other diseases in oral cavity and gastrointestinal tract.

Finally, the application of lollipop helps to promote oral health, but does not replace current dental preventive strategies like tooth brushing and fluoride applications provided by dental offices.

\section{Acknowledgements}

This study was supported by C3 Jian Inc, Delta
Dental-California and UCLA Oppenheimer Award.

\section{References}

1 Anon.Oral health in America: a report of the surgeon general. $J$ Calif Dent Assoc 2000; 28: 685-695.

2 DeBiase CB, Austin SL. Oral health and older adults. $J$ Dent Hyg 2003; 77: 125-145.

3 Keyes PH, Jordan HV. Factors influencing the initiation, transmission, and inhibition of dental caries. In: Sognnaes RF, ed. Mechanism of hard tissue destruction. Washington DC: American Association for the Advancement of Science, 1963: 261-283.

4 Huxley HG. The cariogenicity of dietary sucrose at variaous levels in two strains of rat under unrestricted and controlledfrequency feeding conditions. Caries Res 1977; 11: 237-242.

5 Huxley HG. The effect of feeding frequency on rat caries. $J$ Dent Res 1977; 56: 976.

6 Beighton D, Adamson A, Rugg-Gunn A. Associations between dietary intake, dental caries experience and salivary bacterial levels in 12-year-old English schoolchildren. Arch Oral Biol 1996; 41: 271-280.

7 Axelsson P. Diagnosis and risk prediction of dental caries. Vol. 2. Illinois: Quintessence Pub. Co., 2000.

8 Loesche WJ. Role of Streptococcus mutans in human dental decay. Microbiol Rev 1986; 50: 353-380.

9 Bowden GHW. Which bacteria are cariogenic in humans? In: Johnson NW, ed. Risk markers for oral disease: dental caries. Vol. 1. Cambridge: Cambridge University Press, 1991: 266286.

10 van Houte J. Role of micro-organisms in caries etiology. $J$ Dent Res 1994; 73: 672-681.

11 Mandel ID. Dental caries. Am Sci 1979; 67: 680-688.

12 Hicks J, Garcia-Godoy F, Flaitz C. Biological factors in dental caries: role of saliva and dental plaque in the dynamic process of demineralization and remineralization (part 2). $J$ Clin Pediatr Dent 2004; 28: 119-124.

13 Hicks J, Garcia-Godoy F, Flaitz C. Biological factors in dental caries: role of saliva and dental plaque in the dynamic process of demineralization and remineralization (part 3). $J$ Clin Pediatr Dent 2004; 28: 203-214.

14 Forsten L, Karjalainen S. Effect of a $\mathrm{Ca}(\mathrm{OH})_{2}$ solution and a chlorhexidine based detergent on the microbial activity of human carious teeth. Acta Odontol Scand 1977; 35: 275-280.

15 He J, Heber D, Shi W. Antibacterial compounds from Glycyrrhiza uralensis. J Nat Prod 2006; 69: 121-124.

16 Maron DM, Ames BN. Revised methods for the Salmonella mutagenicity test. Mutat Res 1983; 113: 173-215.

17 Shi W, Jewett A, Hume WR. Rapid and quantitative detection of Streptococcus mutans with species-specific monoclonal antibodies. Hybridoma 1998; 17: 365-371.

18 Ody P. The complete guide medicinal herbal. Vol. 2. London: 
Dorling Kindersley, 2000.

19 Isbrucker RA, Burdock GA. Risk and safety assessment on the consumption of Licorice root (Glycyrrhiza sp.), its extract and powder as a food ingredient, with emphasis on the pharmacology and toxicology of glycyrrhizin. Regul Toxicol Pharmacol 2006; 46: 167-192.
20 Loesche WJ. Dental caries: a treatable infection. Springfield: Charles C Thomas Publisher, 1982.

21 Takahashi I, Okahashi N, Matsushita K, et al. Immunogenicity and protective effect against oral colonization by Streptococcus mutans of synthetic peptides of a streptococcal surface protein antigen. J Immunol 1991; 146: 332-336. 